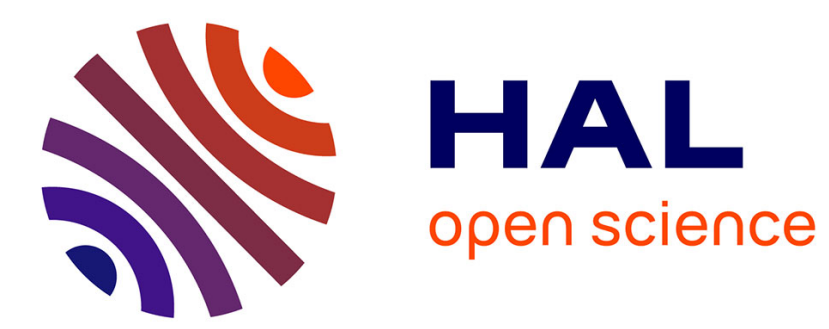

\title{
TECHNIQUE DE L'ÉPREUVE DE LA PHOSPHATASE
}

H. D. Kay, F. K. Neave

\section{To cite this version:}

H. D. Kay, F. K. Neave. TECHNIQUE DE L'ÉPREUVE DE LA PHOSPHATASE. Le Lait, 1938, 18 (180), pp.1041-1049. hal-00895343

\section{HAL Id: hal-00895343 \\ https://hal.science/hal-00895343}

Submitted on 1 Jan 1938

HAL is a multi-disciplinary open access archive for the deposit and dissemination of scientific research documents, whether they are published or not. The documents may come from teaching and research institutions in France or abroad, or from public or private research centers.
L'archive ouverte pluridisciplinaire HAL, est destinée au dépôt et à la diffusion de documents scientifiques de niveau recherche, publiés ou non, émanant des établissements d'enseignement et de recherche français ou étrangers, des laboratoires publics ou privés. 


\title{
BIBLIOGRAPHIE
}

[1] Boutaric. Les colloïdes, Alean, 1931.

[2] Babonneix. Les régimes chez l'enfant, Masson et Cie, 1936 (Bibliographie détaillée).

[3] Вонм. Nourrisson, 1936-1937.

[4] Brennermann. Journal of Amer. Med. Ass., 1929.

[5] Cassie-Cox. Zbl. f. Kinderhk, 1927, 135.

[6] Ferr. Die Sauermileharten, Basel, Schwabe et Co., 1937.

[7] Kleebere-Behrendt. Die Nährpräparate und Sauermilcharten, Enke Verlag, 1930 (Bibliographie détaillée).

[8] LeSNÉ-DreyfuSS. Le concours médical, mai 1934.

[9] Lindet. Le Lait, 1925, p. 953.

[10] Marfan. Nourrisson, 1919, 321 ; 1937.

[11] J. V. Masquet. L'emploi des laits acides chez le nourrisson, Thèse, Paris, 1928 .

[12] R. Mathieu. Encyclopédie Médico-Chirurgicale, Pédiatrie, livre VIII, $4022,4051$.

[13] Monquio. Amer. Journal Dis. Childr., II, 1931, 421.

[14] Mourtquand. Précis de diététique, Testut, 1926.

[15] Pien-Martin. Le Lait, 1932, 513.

[16] L. Prk Ler, Le Lait, 1938, p. 681-698 (Bibliographie détaillée). Nourrisson, 1938 , p. 312 ; Gazette des Hôpitaux, N०78, 1938.

[17] Ch. Porcher. Le Lait, 1932, p. 998.

[18] D. Ronget. Bulletin médical, 1935, 30.

[19] Rossi. Le Lait, $n^{\circ} 93,1930$.

[20] TAPERnOUx. Le Lait, 1928, p. 686, 795, 916.

[21] F. VLès. Précis de Chimie-Physique, Vigot, 1929.

\section{TECHNIQUE DE L'ÉPREUVE DE LA PHOSPHATASE (1)}

\section{par}

\author{
le Professeur H. D. KAY et F. K. NEAVE
}

o. B. E., Ph. D., D. Sc. et B. S. A. National Institute for Research in Dairying

Depuis que les détails de l'épreuve de la phosphatase destinée à contrôler l'efficacité de la pasteurisation furent publiés pour la première fois (2) (mai 1935), sa technique a subi quelques légères modifications. L'expérience a encore démontré la valeur de l'épreuve comme moyen de contrôle du fonctionnement régulier des usines de pasteurisation. Nous avons reçu de nombreuses demandes de renseignements de personnes et d'associations appartenant à l'industrie de la laiterie, concernant les détails de la technique de cette épreuve, et nous avons cru qu'il y aurait intérêt à rassembler tous les renseignements la concernant dans un article qui serait publié dans un

(1) Dairy Industries, janvier 1937 (Traduction R. N. GöRANsson).

(2) Kay H. D. et GRaham W. R., Journal Dairy Research, 6, 1935, 191. 
journal très répandu parmi les industriels, qui s'occupent de lapasteurisation du lait.

L'épreuve est basée sur les faits suivants : a) l'enzyme nommée phosphatase est toujours présente dans le lait cru et est, pour ainsi dire, complètement détruite par une pasteurisation adéquate, e'est. à-dire la pasteurisation effectuée selon les preseriptions du Ministère de l'Hygiène ; $b$ ) la phosphatase, même en petites quantités, est facilement décelable par une réaction chimique relativement simple. L'absence de cette enzyme dans du lait chauffé indique que le chauffage a été adéquat, tandis que sa présence révèle un ehauffage inadéquat ou l'addition de lait cru au lait chauffé. La phosphatase est facilement décelée par sa faculté d'hydrolyser les esters phosphoriques dans certaines conditions; l'épreuve de la phosphatase est basée sur le fait que l'enzyme libère du phénol d'une de ses combinaisons avec l'acide phosphorique. Une petite quantité de lait additionnée d'une solution de phénylphosphate disodique est chauffée, et la quantité de l'enzyme présente, qui indique le degré de défectuosité de la pasteurisation, est mesurée par la détermination colorimétrique de la quantité de phénol libérée en un temps donné.

\section{Outillage requis.}

1. Dans la majorité des cas, un simple comparateur (1) avec un ou plusieurs verres colorés standard Lovibond (2) constitue un instrument de mesure suffisant.

Si on dispose d'un tintomètre Lovibond les couleurs produites au cours de l'épreuve peuvent être exactement déterminées. Dans ce cas, il importe d'utiliser une chambre de $\mathbf{1 3}$ millimètres afin de pouvoir coordonner les données observées avec celles de Kay et Graham.

2. Des papiers filtres Whatman de 9 centimètres $\mathrm{n}^{\circ} 30$ ou $\mathrm{n}^{\circ} 42$, de préférence les derniers.

3. Un bain d'eau dont la température peut être maintenue à $47^{\circ} \mathrm{C} . \pm 2^{\circ} \mathrm{C}$.

4. Un bain d'eau ou une étuve à température maintenue à $37-38^{\circ} \mathrm{C}$.

5. Des pipettes de $0 \mathrm{~cm}^{3} 5$, qualité A ou N.P.L. de préférence. Une pipette séparée est exigée pour chaque échantillon de lait.

En plus, il faut encore quelques burettes pour déterminer les quantités requises des solutions utilisées, une réserve abondante d'éprouvettes à diamètre de $5 / 8 \mathrm{inch}(1 \mathrm{~cm} 5)$ marquées à $10 \mathrm{~cm}^{3}$, avec des bouchons de caoutchoue et des entonnoirs de filtration en verre.

(1) Fourni par "Tintometer Ltd. Salisbury".

(2) La couleur produite dans les conditions de l'épreuve n'est pas un bleu vrai, mais contient un peu de rouge et de jaune. Les verres colorés standard donnent les teintes requises. 


\section{Réactifs requis.}

1. Des comprimés de substrat tampon (1). Un comprimé doit être dissous dans $50 \mathrm{~cm}^{3}$ d'eau distillée, saturée de chloroforme pur. (Si la solution n'est pas préparée fraîchement chaque fois, elle doit être conservée en frigorifère, additionnée d'une ou deux gouttes de chloroforme en plus).

2. Le réactif au phénol de Folin et Ciocalteu (1). Avant de l'utiliser, on le dilue de deux fois son volume d'eau distillée. Ce réactif doit être maintenu à l'abri de la poussière, de métaux, etc., et de n'importe quelle substance réductrice.

3. Une solution à $14 \%$ de carbonate de sodium pur anhydre.

Anderson, Herschdorfer et Neave (2) ont démontré que le substrat tampon est stable pendant plusieurs mois dans ces conditions, et que le réactif de Folin et Ciocalteu est stable pendant au moins quatre mois.

\section{Technique des épreuves.}

Deux épreuves sont recommandées, A et B. La première épreuve A ne prend qu'une demi-heure, elle convient pour déterminer qu'il y a eu chauffage du lait ou non et pour déceler des défauts graves de la technique de la pasteurisation. L'épreuve B convient tant pour les défauts légers que pour les défáuts graves de la pasteurisation, elle est d'application générale au laboratoire de contrôle.

Lorsque ces deux épreuves sont appliquées dans les conditions décrites ci-dessous, le lait adéquatement pasteurisé donne une couleur bleue pâle, qui ne dépasse pas 2,3 unités (Lovibond), jugée dans les conditions standard dans un tube de 13 millimètres ou lorsqu'elle est comparée aux verres standard dans le comparateur spécial.

\section{Technique de l'épreuve A (épreuve brève).}

1. Dans une éprouvette de $25 \mathrm{~cm}^{3}$ on ajoute à $10 \mathrm{~cm}^{3}$ du substrattampon, $0 \mathrm{~cm}^{3} 5$ de lait bien mélangé, on mélange bien et on place pendant 10 minutes dans le bain-marie à la température de $47 \pm 2^{\circ} \mathrm{C}$.

2. On enlève l'éprouvette du bain et on la refroidit à $15-20^{\circ} \mathrm{C}$. par immersion dans de l'eau froide.

3. On ajoute $4 \mathrm{~cm}^{3} 5$ du réactif de Folin et Ciocalteu dilué, on laisse reposer pendant 3 minutes et on filtre.

4. A $10 \mathrm{~cm}^{3}$ du filtrat, prélevés immédiatement après la filtration, on ajoute $2 \mathrm{~cm}^{3}$ de la solution de carbonate de sodium, on mélange bien et on place dans de l'eau bouillante, qui est maintenue en ébullition pendant 5 minutes, puis on filtre de nouveau.

(1) Fournis par "The British Drag Houses Limited n.

(2) Analyst, 1937. 
Cette épreuve doit être faite en double et, en même temps, on fait en double, une épreuve témoin, de la façon suivante :

1. On mélange bien $10 \mathrm{~cm}^{3} \mathrm{du}$ substrat-tampon avec $4 \mathrm{~cm}^{3} 5 \mathrm{du}$ réactif de Folin et Ciocalteu et $0 \mathrm{~cm}^{3} 5$ de lait, on laisse reposer pendant 3 minutes et on filtre.

2. A $10 \mathrm{~cm}^{3}$ du filtrat on ajoute $2 \mathrm{~cm}^{3}$ de la solution de carbonate de sodium, on mélange bien et on place le tube contenant le mélange dans de l'eau bouillante, qui est maintenue en ébullition pendant 5 minutes et on filtre de nouveau.

3. On compare les couleurs des quatre tubes avec celles des verres standard.

\section{Interprétation des résultats de l'épreuve $\mathbf{A}$.}

Si on obtient une couleur bleu pâle dans chacune des quatre éprouvettes, le lait a été chauffé. Si dans les tubes témoins on obtient plus qu'une trace de couleur bleue et si les réactifs ne contiennent pas de phénol libre, il est probable qu'un organisme producteur de phénol est présent dans le lait. Cela ne se présente pas pour du lait pasteurisé qui a été conservé à une température assez basse pour assurer une bonne conservation. Avec du lait frais, pasteurisé d'une façon adéquate, done du lait refroidi à $55^{\circ} \mathrm{F}$. ( $12^{\circ} 7 \mathrm{C}$.) immédiatement après la pasteurisation, et maintenu entre cette température et celle de $65^{\circ} \mathrm{F} .\left(18^{\circ} 3 \mathrm{C}\right.$.) pendant pas plus de 18 heures, les tubes témoins ne peuvent prendre, au plus, qu'une très faible couleur bleue. Lorsqu'avec de tels tubes témoins, on constate dans les tubes, qui ont subi l'incubation, une couleur bleue nette, c'est-à-dire d'une valeur supérieure à 2,3 unités, on peut affirmer que le lait n'a pas subi une pasteurisation adéquate, et lorsque la couleur bleue dépasse 6,0 unités, il est probable que le lait n'a pas été chauffé du tout. Lorsqu'on aura acquis quelque expérience, il est possible qu'on n'aura plus besoin d'éprouvettes témoins pour la majorité des échantillons, auxquels on appliquera cette épreuve.

Si la production d'une couleur bleue plus intense que la couleur standard peut être considérée comme une preuve de pasteurisation inadéquate ou d'addition de lait cru au lait pasteurisé, l'inverse n'est pas toujours vrai. Par exemple, lorsque le chauffage a eu lieu à $140^{\circ}$ F. $\left(60^{\circ}\right.$ C.), au lieu de la température réglementaire de $145^{\circ}$ F. ( $62^{\circ} 8$ C.) ou à une température supérieure, la couleur obtenue avec l'épreuve A ne sera pas plus prononcée que la couleur standard. Dans les cas où la couleur obtenue ne présentera qu'une très légère différence avec la couleur standard, ou lorsque des indications plus précises concernant l'efficacité de la pasteurisation sont nécessaires, ou, d'une façon générale, lorsqu'on en a le temps, on appliquera l'épreuve B qui est plus précise. 


\section{Technique de l'épreuve B.}

1. Dans une éprouvette bouchée de $25 \mathrm{~cm}^{3}$, on ajoute à $10 \mathrm{~cm}^{3}$, du substrat-tampon, $0 \mathrm{~cm}^{3} 5$ du lait bien mélangé et on mélange bien.

2. On ajoute 3 gouttes de chloroforme et après avoir fermé l'éprouvette on la maintient pendant 24 heures à $37-38^{\circ} \mathrm{C}$.

3. A la fin de cette période d'incubation, on refroidit et on ajoute $4 \mathrm{~cm}^{3} 5$ du réactif de Folin et Ciocalteu, on mélange, on laisse reposer pendant 3 minutes, puis on filtre.

4. A $10 \mathrm{~cm}^{3}$ du filtrat, on ajoute $2 \mathrm{~cm}^{3}$ de la solution de carbonate de sodium, on mélange bien et on place dans de l'eau bouillante, on maintient à l'ébullition pendant 5 minutes et on filtre de nouveau.

5. On compare la couleur du filtrat dans le tintomètre ou dans le comparateur. Les épreuves doivent toujours être faites en double et des épreuves témoins doivent être faites, si nécessaire. Pour l'épreuve B, les épreuves témoins doivent être achevées en quelques minutes comme pour l'épreuve A, où la filtration doit être faite après l'addition du réactif de Folin et Ciocalteu et le filtrat doit être conservé au froid jusqu'au lendemain. Dans la pratique, on peut également conserver les échantillons de lait au froid pendant 24 heures, lorsque les deux éprouvettes contenant le substrat-tampon et le lait ont été placées à l'étuve à $27-38^{\circ} \mathrm{C}$, et ne faire les épreuves témoins que le lendemain, pour les échantillons, qui fournissent une réaction positive après 24 heures d'incubation.

\section{Interprétation des résultats de l'épreuve $\mathbf{B}$.}

Lorsque la couleur produite est plus prononcée que celle du verre standard 2,3, la pasteurisation du lait a été inadéquate ; c'est-à-dire ou a) la température de pasteurisation n'a pas été assez élevée, ou b) la durée du chauffage à la température de pasteurisation a été trop brève, ou c) une petite quantité de lait cru a été mélangée au lait pasteurisé.

Le mélange de $0,2 \%$ de lait cru à un échantillon de lait adéquatement pasteurisé fournit un produit, qui examiné par cette méthode donna une couleur bleue égale à 2,5 unités, tandis que l'addition de $0,5 \%$ de lait cru eut pour effet une hausse de la valeur du bleu à 3,7 unités dans un cas, à 4,2 unités dans un autre cas. Ces résultats autorisent de conclure que cette méthode permet de déceler facilement le mélange de $0,5 \%$ de lait cru à du lait pasteurisé.

Des observations plus récentes ont démontré que, dans la pratique, on parvient facilement à déceler le mélange de $0,2 \%$ de lait eru à du lait adéquatement pasteurisé.

La couleur du filtrat des épreuves témoins ne peut dépasser la valeur de 1,5 unité de bleu.

Du lait, dont les épreuves donnent moins de 1,5 unités, et qui 
donne une couleur de valeur inférieure à 2,3 unités de bleu, peut être considéré adéquatement pasteurisé. Les laits qui donnent des couleurs entre 2,3 et 6,0 unités de bleu doivent être considérés inadéquatement pasteurisés, tandis que lorsqu'on obtient des couleurs

\begin{tabular}{|c|c|c|c|c|c|}
\hline$N^{\circ}$ & $\begin{array}{l}\text { Mois } \\
1936\end{array}$ & $\begin{array}{c}\text { Impor- } \\
\text { tance } \\
\text { de } \\
\text { l'usine }\end{array}$ & $\begin{array}{c}\text { Nature } \\
\text { de l'échantillon }\end{array}$ & $\begin{array}{c}\text { Résultat } \\
\text { numérique } \\
\text { de } \\
\text { l'épreuve } \\
\text { (Unités } \\
\text { de bleu) }\end{array}$ & Conclusion \\
\hline 1 & juin & grande & 1/3 pinte (école) & 1,2 & pasteurisé. \\
\hline 2 & juin & moyenne & 1/3 pinte (école) & 1,5 & pasteurisé. \\
\hline 3 & juin & petite & $1 / 3$ pinte (école) & 1,3 & pasteurisé. \\
\hline 4 & & & chambreur 4 & $30+$ & $\begin{array}{l}\text { chauffage très défec- } \\
\text { tueux (1). }\end{array}$ \\
\hline 5 & juin & grande & chambreur 1 & $30+$ & $\begin{array}{l}\text { chauffage très défec- } \\
\text { tueux. }\end{array}$ \\
\hline 6 & & & chambreur 2 & 5,2 & $\begin{array}{l}\text { pasteurisation inadé- } \\
\text { quate. }\end{array}$ \\
\hline 7 & juillet & moyennel & 1 pinte & 2,3 & pasteurisé. \\
\hline 8 & juillet & grande & $1 / 3$ pinte (école) & 1,1 & pasteurisé. \\
\hline 9 & sept. & grande & $1 / 3$ pinte (école) & 1,1 & pasteurisé. \\
\hline 10 & sept. & petite & $1 / 3$ pinte (école) & 1,4 & pasteurisé. \\
\hline 11 & oct. & grande & 1/3 pinte (école) & 1,5 & pasteurisé. \\
\hline 12 & oct. & moyenne & chambreur & 1,1 & pasteurisé. \\
\hline 13 & oet. & petite & échantillon flacon & $30+$ & $\begin{array}{l}\text { chauffage très défec- } \\
\text { tueux ou lait cru. }\end{array}$ \\
\hline 14 & oct. & petite & échantillon flacon & 1,3 & pasteurisé. \\
\hline 15 & oct. & petite & échantillon flacon & 1,3 & pasteurisé. \\
\hline 16 & oct. & moyenne & $1 / 3$ pinte (école) & 6,4 & $\begin{array}{l}\text { chauffage très défec- } \\
\text { tueux. }\end{array}$ \\
\hline 17 & oct. & grande & 1/3 pinte (école) & 1,6 & pasteurisé. \\
\hline 18 & oct. & petite & 1/3 pinte (école) & 1,5 & pasteurisé. \\
\hline 19 & oct. & moyenne & bouteille 1 pinte & 1,3 & pasteurisé. \\
\hline 20 & oet. & moyenne & bouteille 1 pinte & 5,0 & $\begin{array}{l}\text { pasteurisation inadé- } \\
\text { quate. }\end{array}$ \\
\hline 21 & oct. & moyennel & bouteille 1 pinte & 1,2 & pasteurisé. \\
\hline 22 & oct. & moyenne & $\{$ ehambreur 1 & 7,8 & $\begin{array}{l}\text { chauffage très défec- } \\
\text { tueux (2). }\end{array}$ \\
\hline 23 & & & chambreur 3 & 1,2 & pasteurisé. \\
\hline 24 & oet. & moyenne & bouteille $1 / 2$ pinte & 3,5 & $\begin{array}{l}\text { pasteurisation inadé- } \\
\text { quate. }\end{array}$ \\
\hline
\end{tabular}

(1) Usine à outillage défectueux.

(2) Fonctionnement défectueux d'un chambreur. 
plus prononcées que 6,0 on peut affirmer qu'il y a eu de graves défauts techniques. Le lait cru donne généralement plus de 30 unités de couleur.

Le tableau ci-devant donne des résultats typiques de l'épreuve de la phosphatase (épreuve B) obtenus récemment avec du lait vendu au public ou destiné à être mis en vente.

\section{Limites des indications fournies par l'épreuve B.}

Il n'est pas possible de déterminer seulement par l'épreuve de la phosphatase quelle est la cause spécifique d'un chiffre élevé. Tout ce qu'on peut aff rmer, c'est que la pasteurisation du lait est inadéquate. Les défauts les moins graves, qui peuvent être décelés par l'épreuve $\mathrm{B}$ appliquée conformément à la description donnée ici, sont les suivants: (1)

a) Le chambrage à $145^{\circ} \mathrm{F}$. ( $62^{\circ} 8 \mathrm{C}$.) pendant 20 au lieu de 30 minutes.

b) Le chambrage à $101 / 2 \mathrm{~F}$. en dessous de la température minima prescrite (c'est-à-dire à $143^{\circ} \mathrm{F}$. (61094 C.) ou en dessous).

c) L'addition de $0,2 \%$ de lait cru à du lait adéquatement pasteurisé.

\section{Précautions à prendre.}

Si possible, il faut appliquer l'épreuve moins de 18 heures après la pasteurisation.

Anderson, Herschdorfer et Neave (2) ont démontré eependant que des échantillons de lait peuvent être conservés en resserre froide plusieurs jours, - même pendant une quinzaine, - sans influence sur les résultats de l'épreuve de la phosphatase.

On doit tenir les savons à l'acide phénique, le phénol ou les désinfectants de ce type à distance des endroits où des épreuves sont appliquées.

La verrerie doit être soigneusement nettoyée au moyen d'une solution bouillante de sel de soude, avant l'utilisation. On ne peut utiliser du savon. désinfectant.

\section{Application à du lait soumis à la pasteurisation brève à haute température.}

Dans deux séries de nombreuses expériences, dans une desquelles fut utilisé du lait pasteurisé dans un appareil Stassano (lait chauffé pendant 15 à 20 secondes à $167^{\circ} \mathrm{F}$. $\pm 0^{\circ} 5 \mathrm{~F}$. $\left(75^{\circ} \mathrm{C} . \pm 0^{\circ} 27 \mathrm{C}\right.$.) dans l'autre, du lait pasteurisé dans un appareil A.P.V. à plaques

(1) Avec des modifications spéciales, des défauts moins importants peuvent être décelés.

(2) Analyst, 1937. 
pour le même type de chauffage. La pasteurisation fut faite dans les conditions requises, et aucun des échantillons ne donna une réaction positive avec l'épreuve de la phosphatase.

Dans d'autres expériences avec un pasteurisateur industriel, du lait chauffé à $155^{\circ} \mathrm{F}$. $\left(68,3^{\circ} \mathrm{C}\right.$.) pendant 10 secondes, sans autre chauffage, donna un résultat positif, même avec l'épreuve A. Le lait chauffé dans le même appareil à $160^{\circ} \mathrm{F}$. $\left(71^{\circ} 1 \mathrm{C}\right.$.) pendant 10 secondes, sans autre chauffage donna une réaction positive avec l'épreuve $B$, mais une réaction négative avec l'épreuve $\mathrm{A}$. Il est évident que l'épreuve de la phosphatase peut également servir au contrôle de l'efficacité de la pasteurisation brève à température élevée. Des observations antérieures ont démontré que la phosphatase est détruite, dans une région de températures et de durées de chauffage étendue, relativement moins facilement que le Mycobacterium tuberculosis, le plus résistant à la chaleur des organismes pathogènes communs.

\section{Application à d'autres produits de laiterie.}

a) Lait homogénéisé. - L'épreuve de la phosphatase peut être appliquée directement à du lait homogénéisé.

b) Crème. - La meilleure façon est de diluer la crème au moyen d'eau jusqu'à une teneur de matière grasse de $4-6 \%$, puis de procéder comme pour le lait.

c) Beurre. - Prélever 30 grammes de beurre dans un tube de centrifugation de $100 \mathrm{~cm}^{3}$, laisser fondre à $40^{\circ} \mathrm{C}$., centrifuger et enlever la couche aqueuse au moyen d'une pipette.

Pour l'épreuve A, prendre $1 / 2 \mathrm{~cm}^{3}$ de cette couche aqueuse au lieu de $1 / 2 \mathrm{~cm}^{3}$ de lait. Chauffer pendant 10 minutes à $47^{\circ} \mathrm{C}$, refroidir et procéder comme pour du lait. Un bleu plus prononcé que le standard 2,3 unités de bleu indique de la crème crue ou à pasteurisation défectueuse. Confirmation a été obtenue avec du beurre préparé de erème crue, de crème pasteurisée au laboratoire par le maintien à $145^{\circ}$ F. ( $62^{\circ} 8$ C.) pendant une demi-heure, et avec du beurre de Nouvelle-Zélande fait de crème crue et de crème pasteurisée par la méthode de chauffage rapide en usage dans ce pays.

Dans ce dernier cas, des résultats négatifs furent obtenus, tandis que les beurres faits de crème crue, même après conservation pendant 6 semaines à de basses températures, donnèrent des réactions positives nettes.

Une autre méthode, qui donne également satisfaction et qui est peut être moins compliquée, consiste à faire fondre environ 20 grammes de beurre additionnés de $10 \mathrm{~cm}^{3}$ d'eau (ou 40 grammes de beurre et $20 \mathrm{~cm}^{3}$ d'eau) dans une éprouvette. Après fusion à $40^{\circ} \mathrm{C}$., bien agiter, boucher l'éprouvette et mettre au réfrigérateur, jusqu'après 
solidification de la matière grasse. Appliquer l'épreuve A, en utilisant $0 \mathrm{~cm}^{3} 5$ de la couche aqueuse.

Lorsque le beurre n'est pas plus vieux que deux semaines, l'épreuve de la phosphatase peut servir à déterminer si le beurre a été fait de crème crue ou de crème pasteurisée.

d) Fromage. - Des expériences sont en cours en vue de l'application de l'épreuve au fromage. Dans le fromage fait de lait cru, de notables quantités de phosphatase sont présentes pendant deux mois au moins après la fabrication.

\title{
REVUE
}

\section{UNE IMÉTHODE PRÉCISE POUR LA DÉTERMINATION DU CAROTENNE DANS LE FOURRAGE}

\author{
par G. GENIN
}

Ingénieur-Chimiste E. P. C.

On sait que le carotène est un des constituants parmi les p̂lus importants du fourrage par l'influence qu'il exerce sur la composition du lait et du beurre qui en est extrait. Il n'est done pas étonnánt que l'on ait cherché à mettre au point des méthodes simples et rapides de dosage de ce produit. Munser [1] a publié récemment une étude sur les méthodes décrites pour ce dosage ; mais toutes ces méthodes ne donnent de bons résultats que si des précautions tout à fait spéciales sont prises. La méthode de GuILBERT [2] donne également de bons résultats, toutefois, elle a l'inconvénient d'imposer l'emploi d'éther éthylique pour l'extraction du carotène, du mélange saponifié. La modification apportée à cette méthode par Peterson et Hughes [3] supprime l'emploi de l'éther éthylique et le carotène est extrait directement du mélange saponifié au moyen d'éther de pétrole, malheureusement, il y a, dans ce cas, formation d'émulsions et perte de carotène.

Il nous a done semblé utile d'indiquer une nouvelle modification apportée à ce mode opératoire et due à D. W. Bolrn et A. M. KHALAPAR, modification qui a été employée avec succès pendant plusieurs années dans les laboratoires de ces auteurs [4]. Le nouveau procédé a l'avantage, par l'emploi de quantités définies de réactif, d'éviter la formation d'émulsion et de donner des résultats plus précis.

\section{Mode opératoire.}

On traite, au reflux, un échantillon de 5 à 10 grammes pendant 40 minutes avec $200 \mathrm{~cm}^{3}$ d'alcool éthylique à $95-97 \%$. On filtre 